\title{
ORIGINAL ARTICLE \\ Homoeologous chromosomes of Xenopus laevis are highly conserved after whole-genome duplication
}

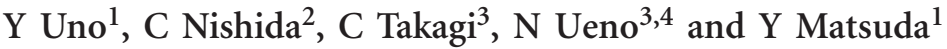

It has been suggested that whole-genome duplication (WGD) occurred twice during the evolutionary process of vertebrates around 450 and 500 million years ago, which contributed to an increase in the genomic and phenotypic complexities of vertebrates. However, little is still known about the evolutionary process of homoeologous chromosomes after WGD because many duplicate genes have been lost. Therefore, Xenopus laevis $(2 n=36)$ and Xenopus (Silurana) tropicalis $(2 n=20)$ are good animal models for studying the process of genomic and chromosomal reorganization after WGD because $X$. laevis is an allotetraploid species that resulted from WGD after the interspecific hybridization of diploid species closely related to $X$. tropicalis. We constructed a comparative cytogenetic map of $X$. laevis using 60 complimentary DNA clones that covered the entire chromosomal regions of 10 pairs of $X$. tropicalis chromosomes. We consequently identified all nine homoeologous chromosome groups of $X$. laevis. Hybridization signals on two pairs of $X$. laevis homoeologous chromosomes were detected for 50 of $60(83 \%)$ genes, and the genetic linkage is highly conserved between $X$. tropicalis and $X$. laevis chromosomes except for one fusion and one inversion and also between $X$. laevis homoeologous chromosomes except for two inversions. These results indicate that the loss of duplicated genes and inter- and/or intrachromosomal rearrangements occurred much less frequently in this lineage, suggesting that these events were not essential for diploidization of the allotetraploid genome in $X$. laevis after WGD.

Heredity (2013) 111, 430-436; doi:10.1038/hdy.2013.65; published online 3 July 2013
\end{abstract}

Keywords: Xenopus laevis; Xenopus tropicalis; comparative gene mapping; FISH; whole-genome duplication; homoeologous chromosomes

\section{INTRODUCTION}

Whole-genome duplication (WGD: polyploidization) is an evolutionary event that played an important role in the diversification of most eukaryotic lineages (Ohno, 1970; Kellis et al., 2004; Kasahara, 2007; Otto, 2007). It is generally accepted that each duplicated gene evolved independently after WGD and that the polyploid genome quickly turned into a diploid state, referred to as diploidization, through repeated genomic and chromosomal reorganization including the loss of homoeologs, with some genes being consistently maintained as duplicates. Diploidization through genomic and chromosomal reorganization after ancient WGDs has been identified in yeast and most plant genomes (Song et al., 1995; Seoighe and Wolfe, 1998; Pontes et al., 2004; Simillion et al., 2004; Yu et al., 2005; Fischer et al., 2006; Tuskan et al., 2006; Woodhouse et al., 2010). A total of $80 \%$ of the duplicated genes in yeast were lost after WGD 80 million years ago (Mya) (Kellis et al., 2004), 70\% in Arabidopsis after WGD 86 Mya (Bowers et al., 2003) and 47\% in maize after WGD 5-12 Mya (Woodhouse et al., 2010).

Polyploidization is less prevalent in animals than in plants. Comparative analyses of genome sequences in vertebrates and chordates revealed that WGD occurred twice (2R-WGD) in the early evolutionary history of vertebrates around 450 and $500 \mathrm{Mya}$, and
3R-WGD occurred in the teleost fish lineage $>350$ Mya after 2RWGD (Ohno, 1970; International Human Genome Sequencing Consortium, 2001; Jaillon et al., 2004; Kasahara et al., 2007; Putnam et al., 2008). Comparative genome analyses of four teleost fish species (medaka fish, Takifugu, Tetraodon and zebrafish) suggested that genomic and chromosomal reorganization frequently occurred in the teleost fish lineage after 3R-WGD (Kasahara et al., 2007; Sémon and Wolfe, 2007). It has been suggested that the common ancestor of salmonid fishes underwent one additional WGD 50-100 Mya after 3R-WGD (Allendorf and Thorgaard, 1984). Comparisons of genetic linkage between salmonid fishes (Atlantic salmon and rainbow trout) and other teleost fishes (medaka, stickleback and zebrafish) revealed that the genetic linkage of teleost fishes is conserved in salmonid fishes, although several chromosomal rearrangements occurred after WGD in salmonid genomes (Danzmann et al., 2008; Guyomard et al., 2012). However, little is known about the process of genomic and chromosomal reorganization after WGD in vertebrates because many duplicate genes derived from WGD have been lost in vertebrate genomes. For example, genome sequence analyses revealed that $76-80 \%$ of duplicated genes derived from 3RWGD have been lost in extant teleost fish lineages, and it is speculated that approximately $60 \%$ of these duplicated genes were rapidly lost

${ }^{1}$ Laboratory of Animal Genetics, Department of Applied Molecular Biosciences, Graduate School of Bioagricultural Sciences, Nagoya University, Nagoya, Japan; ${ }^{2}$ Department of Natural History Sciences, Faculty of Science, Hokkaido University, Sapporo, Japan; ${ }^{3}$ Division of Morphogenesis, National Institute for Basic Biology, Okazaki, Japan and ${ }^{4}$ Department of Basic Biology, School of Life Science, The Graduate University of Advanced Studies (SOKENDAI), Okazaki, Japan

Correspondence: Dr Y Matsuda, Laboratory of Animal Genetics, Department of Applied Molecular Biosciences, Graduate School of Bioagricultural Sciences, Nagoya University, Nagoya 464-8601, Japan.

E-mail: yoimatsu@agr.nagoya-u.ac.jp

Received 17 January 2013; revised 6 May 2013; accepted 9 May 2013; published online 3 July 2013 
within about 75 million years after 3R-WGD (Jaillon et al., 2004; Sato et al., 2009).

The African clawed frog (Xenopus laevis, Pipidae, Anura) $(2 n=36)$ and the western clawed frog (Xenopus (Silurana) tropicalis, Pipidae, Anura) $(2 n=20)$ are widely used as experimental animals in a wide range of scientific fields such as developmental, cellular, immunological and molecular biological research. More than 20 species of extant clawed frogs classified into two genera (Silurana and Xenopus) have been reported, which exhibit a variable number of chromosomes $(2 n=20-108)$. It is suggested that these species except for $X$. tropicalis resulted from polyploidization, which occurred after the hybridization of two different species (Tymowska and Fischberg, 1973; Bisbee et al., 1977; Tymowska, 1991; Hughes and Hughes, 1993; Kobel, 1996; Evans et al., 2004, 2008). The allotetraploidization events occurred at least twice in clawed frogs after the divergence of the ancestor of the diploid species $X$. tropicalis, which has 20 chromosomes and the ancestor of diploid species now thought to be extinct, which had 18 chromosomes (Tymowska, 1991; Evans, 2008), and this divergence probably occurred 50-65 Mya (Evans et al., 2004; Hellsten et al., 2007). In Silurana, X. tropicalis and another diploid species underwent allopolyploidization to give rise to three tetraploid species with 40 chromosomes, including $X$. epitropicalis and two undescribed species. Allopolyploidization between two 18-chromosome species occurred at least once 21-40 Mya, giving rise to the ancestor of all Xenopus species with 36, 72 or 108 chromosomes (Evans et al., 2004; Chain and Evans, 2006; Hellsten et al., 2007). Therefore, X. laevis and $X$. tropicalis are good animal models for understanding the process of genomic and chromosomal reorganization after WGD. We recently constructed a high-resolution chromosome map consisting of 140 genes for $X$. tropicalis (Uno et al., 2012). The draft genome assemblies of X. tropicalis were reported previously (Hellsten et al., 2010), and several comparative studies of complimentary DNA (cDNA) sequences have been performed between $X$. tropicalis and X. laevis (Morin et al., 2006; Hellsten et al., 2007; Sémon and Wolfe, 2008). However, the information is still insufficient to know genetic linkage of $X$. laevis, and no comprehensive comparative analyses using genomic sequencing or cytogenetic mapping have been conducted for $X$. laevis and $X$. tropicalis.

In this study, we constructed a comparative cytogenetic map of $X$. laevis by fluorescence in situ hybridization (FISH) mapping of the functional genes that had been localized to $X$. tropicalis chromosomes. We consequently identified all nine homoeologous chromosome groups of X. laevis and then revealed the chromosome rearrangements that occurred between the two species and also between the homoeologous chromosomes of $X$. laevis. We discussed the process of genomic and chromosomal evolution in the X. laevis lineage after WGD on the basis of our comparative cytogenetic map of X. laevis.

\section{MATERIALS AND METHODS}

\section{Animals, cell culture and chromosome preparation}

We used adult females of the J strain of X. laevis, which were purchased from the breeder. After pithing, heart, lung and kidney tissues were collected for cell culture. All experimental procedures using animals conformed to the guidelines established by the Animal Care Committee, Nagoya University. Tissues were minced, and cells were cultured at $26^{\circ} \mathrm{C}$ in a humidified atmosphere of $5 \% \mathrm{CO}_{2}$ in air for 10-14 days (Uno et al., 2008). Primary cultured cells were harvested using $0.5 \%$ trypsin and then subcultured. For replication-banded chromosome preparation, 5-bromo- $2^{\prime}$-deoxyuridine $\left(25 \mu \mathrm{g} \mathrm{ml}^{-1}\right)$ was added to the cell cultures at log phase, and cell culturing was continued for $6 \mathrm{~h}$ including $1 \mathrm{~h}$ colcemid treatment $\left(0.17 \mu \mathrm{g} \mathrm{ml}^{-1}\right)$ before harvesting. Chromosome preparations were made following a standard air-drying method. After staining with Hoechst $33258\left(1 \mu \mathrm{g} \mathrm{ml}^{-1}\right)$ for $5 \mathrm{~min}$, slides were heated to $65^{\circ} \mathrm{C}$ for $3 \mathrm{~min}$ on a hot plate and then exposed to ultraviolet light for an additional $5-6$ min at $65^{\circ} \mathrm{C}$ (Matsuda and Chapman, 1995). Slides were kept at $-80^{\circ} \mathrm{C}$ until use.

\section{Fluorescence in situ hybridization}

For chromosome mapping, $60 \mathrm{X}$. laevis cDNA clones were selected from 140 clones that were used for the chromosome mapping of $X$. tropicalis in our previous study (Uno et al., 2012). These clones were isolated based on a web data catalog of the NIBB/NIG/NBRP Xenopus laevis EST project (XDB3, http:// xenopus.nibb.ac.jp/). FISH mapping was performed as described previously (Matsuda and Chapman, 1995). DNA probes were labeled with biotin16-dUTP (Roche Diagnostics, Basel, Switzerland) using a nick translation kit (Roche Diagnostics) following the manufacturer's instruction and ethanol precipitated with sonicated salmon sperm DNA and Escherichia coli transfer RNA. After hybridization, the hybridized probes were reacted with a goat antibiotin antibody (Vector Laboratories, Burlingame, CA, USA) and then stained with Alexa Fluor 488 rabbit anti-goat IgG $(\mathrm{H}+\mathrm{L})$ conjugate (Molecular Probes, Life Technologies, Carlsbad, CA, USA). Chromosome slides were counterstained with $0.75 \mu \mathrm{g} \mathrm{ml}^{-1}$ propidium iodide. To discriminate the $\mathrm{Z}$ and $\mathrm{W}$ chromosomes, a 15-kb genomic DNA fragment of the $D M-W$ gene (Yoshimoto et al., 2008) was hybridized to the chromosome slide, where the hybridization signal was detected on chromosome 3. The hybridized probe was removed from the slides by redenaturation in $70 \%$ formamide $/ 4 \times$ standard saline citrate at $70{ }^{\circ} \mathrm{C}$ for $2 \mathrm{~min}$, and then the $D M-W$ probe was hybridized with a 100-time volume of the sonicated whole genomic DNA of X. laevis to the same slide. After hybridization, slides were incubated with fluorescein isothiocyanateavidin (Roche Diagnostics), and hybridization signals were observed.

\section{RESULTS}

High-resolution Hoechst-stained bands of X. laevis chromosomes were obtained using a replication banding method (Figure 1), and their ideograms were slightly modified from our previous one (Uno et al., 2008). Based on our previous mapping data of 140 genes in X. tropicalis (Uno et al., 2012), we selected 60 genes, which covered the entire chromosomal regions of 10 pairs of $X$. tropicalis chromosomes, for their comparative mapping to $X$. laevis chromosomes (XLA) (Figure 2 and Table 1). The identification of each chromosome and subchromosomal localization of the hybridization signals was performed using the Hoechst G-banded ideogram. Hybridization signals were detected on two pairs of homoeologous chromosomes for $83 \%$ of the cDNA clones (50/60) (Figure 3). For instance, seven genes (KDM3A, ACSL1, PCDH10, EEF2, DMRT1, NF2 and $D E P D C 1 B)$, which were mapped on $X$. tropicalis chromosome (XTR) 1, were all localized to XLA1 and XLA2, indicating that XLA1 and XLA2 are homoeologous. All the other homoeologous chromosome pairs of $X$. laevis and their homologies with $X$. tropicalis chromosomes were as follows: XTR2 was homologous to XLA3 and XLA8, XTR3 to XLA12 and XLA16, XTR4 to XLA13 and XLA17, XTR5 to XLA4 and XLA5, XTR6 to XLA6 and XLA9, XTR7 to XLA7 and XLA10 and XTR8 to XLA11 and XLA14. The genes on XTR9 and XTR10 were all localized to XLA15 and XLA18, suggesting that the homoeologous chromosome pair XLA15 and XLA18 was derived from a tandem fusion of XTR9 and XTR10 or that XTR9 and XTR10 are derivatives of a fission event that occurred in an original chromosome pair of homoeologous XLA15 and XLA18.

Genetic linkage has been highly conserved between the two species and also between homoeologous chromosome pairs of $X$. laevis. No interchromosomal rearrangements (reciprocal translocations) were detected, and gene orders were identical between homologous chromosomes of $X$. laevis and $X$. tropicalis and between $X$. laevis homoeologous chromosome pairs, except for intrachromosomal rearrangements (inversions) that were detected between XLA12 and XLA16, XLA11 and XLA14, and XTR10 and homoeologous XLA15 


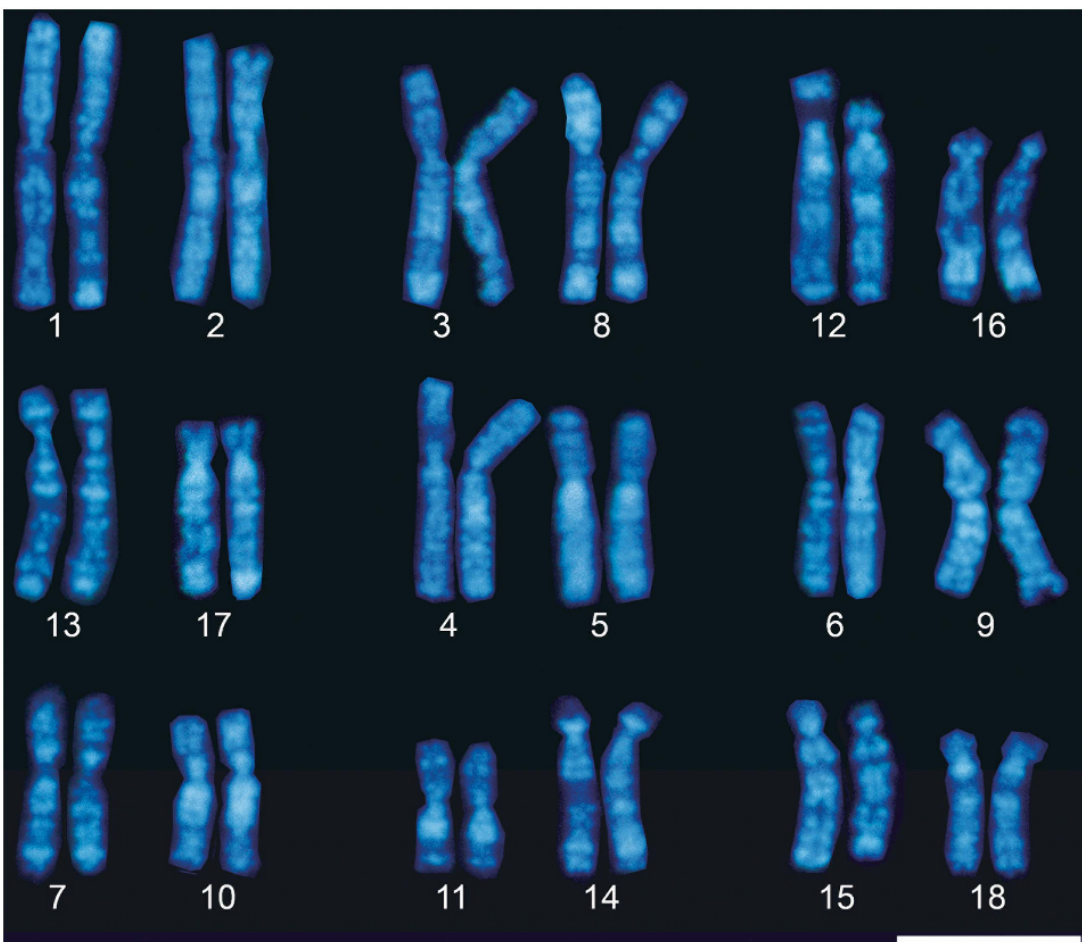

Figure 1 Hoechst-stained replication-banded karyotype of the female $X$. laevis (XLA). Each chromosome was numbered following our previous karyotypic study of $X$. laevis (Uno et al., 2008). Chromosomes were grouped as homoeologous chromosome pairs (XLA1 $+2,3+8,12+16,13+17,4+5,6+9$, $7+10,11+14$ and $15+18$ ) according to comparative gene mapping in this study (Figure 3). Small size variation of chromosomes between individuals was found for XLA12p (Uno et al., 2008), which depends on the size differences of the 18S-28S ribosomal gene cluster on the short arm (data not shown). Scale bar $=10 \mu \mathrm{m}$.

and XLA18 pairs. These results indicate that the genetic linkage of $X$. tropicalis chromosomes has been retained almost intact in X. laevis chromosomes with no interchromosomal translocations after WGD.

Five XTR2-linked genes (POU2F1, EIF2S3, MDH2, LARP4 and $R A B 6 A$ ) were all localized to the homologous chromosomes of XLA3 and XLA8. XLA3 was identified as the Z and $\mathrm{W}$ sex chromosomes in our previous study (Yoshimoto et al., 2008); however, no morphological differences have been found between the $\mathrm{Z}$ and $\mathrm{W}$ sex chromosomes (Schmid and Steinlein, 1991; Uno et al., 2008). We identified the $\mathrm{W}$ chromosome by FISH mapping of the W-linked sex (ovary)-determining gene, $D M-W$ (Yoshimoto et al., 2008) (Figure $2 \mathrm{~m}$ ). None of the five genes on the $\mathrm{Z}$ or $\mathrm{W}$ chromosome differed in chromosomal locations and hybridization efficiencies (data not shown), indicating that structural differentiation hardly occurred between the $\mathrm{Z}$ and $\mathrm{W}$ chromosomes except for the $\mathrm{W}$-specific region containing the $D M-W$ gene.

\section{DISCUSSION}

In this study, we identified all nine quartets (the homoeologous chromosome groups) of X. laevis (XLA1 +2, 3+8, 12+16, 13+17, $4+5,6+9,7+10,11+14$ and $15+18)$, whose genetic linkage has been highly conserved in $X$. tropicalis. Paleontological studies have suggested that $X$. tropicalis is a more ancient species than other extant polyploid Xenopus species, providing the possibility that the ancestral diploid Xenopus species had $2 n=20$ chromosomes similar to $X$. tropicalis (Estes, 1975). Therefore, the chromosome number of $X$. laevis $(2 n=36)$ may have occurred as a result of allotetraploidization of the interspecific hybrid between two different species with $2 n=18$ chromosomes, which was derived from the fusion of two chromosome pairs of the ancestral diploid species with $2 n=20$ (Schmid and Steinlein, 1991; Tymowska, 1991). Our results suggest that the ancestral bi-armed chromosome pair of homoeologous XLA15 and XLA18 may have been derived from the fusion of XTR9 and XTR10 in the ancestral species of X. tropicalis. The nine quartets identified in this study were not consistent with those determined by 5-bromo- 2 -deoxyuridine/dT replication banding (Schmid and Steinlein, 1991) and cross-species chromosome hybridization with $X$. tropicalis-derived chromosome painting probes (Krylov et al., 2010). Krylov et al. (2010) demonstrated that XLA11 + 14 and XLA15 + 18 were painted with XTR8 and XTR9 probes, respectively, and XLA14 and XLA18 were hybridized with XTR10 paint; however, XTR10 showed homology with XLA15 and XLA18 by comparative gene mapping in the present study. This discrepancy may be due to a difference in the numbering system of $X$. laevis chromosomes.

High rate of loss of duplicated genes (50-75\%) after WGD has been reported in $X$. laevis using a large number of expressed sequence tags (ESTs) (20223 ESTs reported by Hellsten et al. (2007) and 28463 ESTs by Sémon and Wolfe (2008)), which is not so different from the rate of gene loss in teleost fishes after 3R-WGD (76-80\%) (Jaillon et al., 2004; Sémon and Wolfe, 2007), yeast after WGD (80\%) (Kellis et al., 2004), Arabidopsis (70\%) (Bowers et al., 2003) and maize (47\%) (Woodhouse et al., 2010). However, in this study, hybridization signals were detected on both homoeologous chromosomes for most clones (50 of 60 genes: 83\%), which indicated that the loss of duplicated genes after WGD was much lower in X. laevis (17\%). These results suggest that the loss of one copy of duplicated genes could not be determined accurately using only a partial collection of 

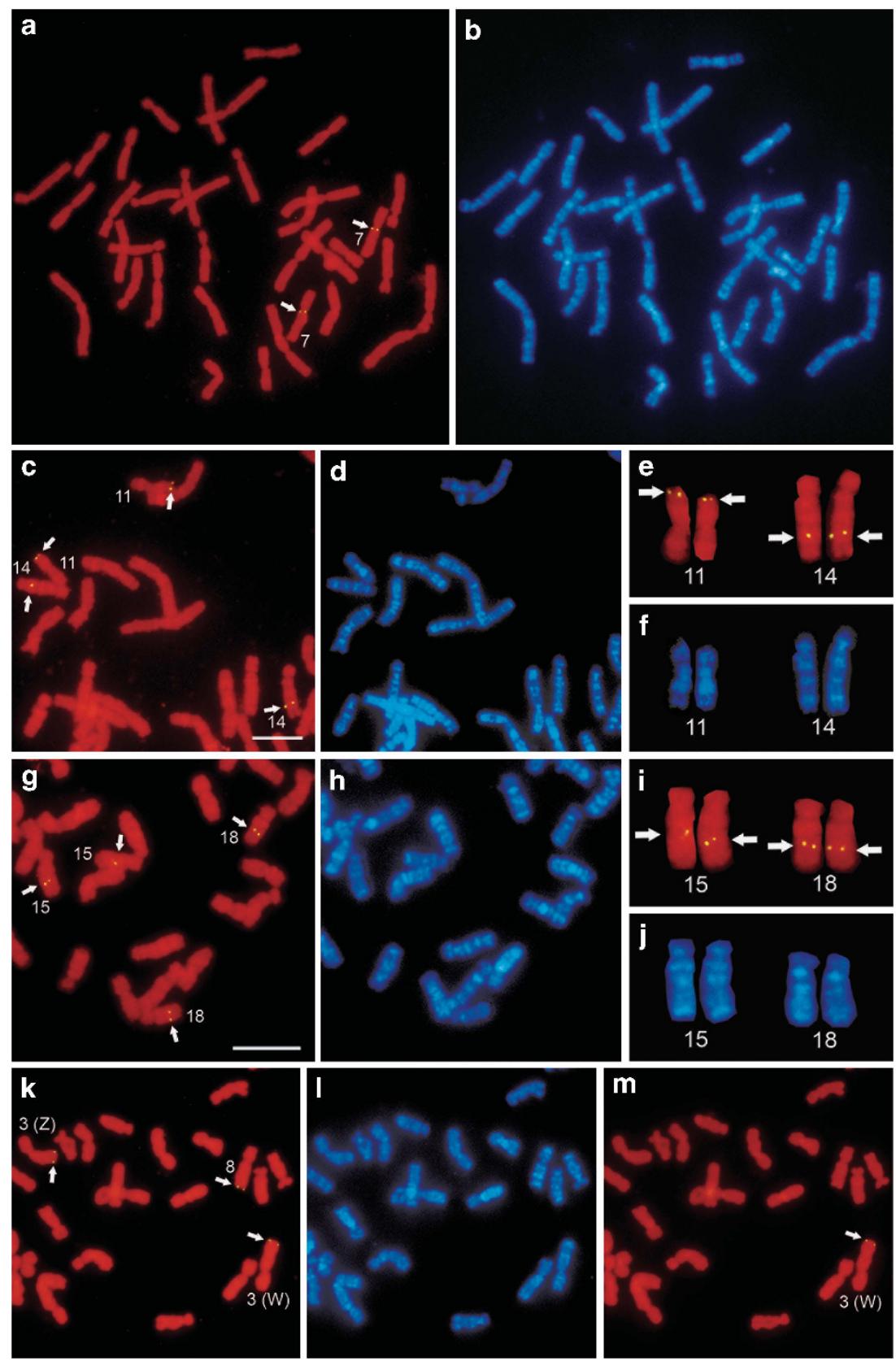

Figure 2 Chromosomal localization of DNA clones to metaphase chromosome spreads of the female $X$. laevis. FISH patterns of the cDNA clones of TUBGCP2 on chromosome 7p (a), ACTN1 on chromosomes $11 \mathrm{p}$ and 14q (c, e), FN1 on chromosomes 15q and 18q (g, i) and RAB6A on chromosomes 3q and $8 \mathrm{q}(\mathbf{k})$ on propidium iodide-stained metaphase chromosome spreads. Arrows indicate hybridization signals. The FISH pattern of a genomic DNA fragment of the $D M-W$ gene on chromosomes $3 q(\mathbf{m})$ on the same metaphase spread that was used for mapping of the $R A B 6 A$ gene $(\mathbf{k})$. The $D M-W$ probe was hybridized to the metaphase spread after the RAB6A probe was removed. The $\mathrm{Z}$ and $\mathrm{W}$ sex chromosomes were precisely identified by FISH with the $D M-W$ probe. Hoechst-stained patterns of the propidium iodide-stained chromosomes in panels $\mathbf{a}, \mathbf{c}, \mathbf{e}, \mathbf{g}, \mathbf{i}$ and $\mathbf{k}$ are shown in panels $\mathbf{b}, \mathbf{d}, \mathbf{f}, \mathbf{h}, \mathbf{j}$ and $\mathbf{I}$, respectively. Scale bars $=10 \mu \mathrm{m}$.

X. laevis gene ESTs (Hellsten et al., 2007). Analyses using both genome sequences and chromosome mapping of a large number of transcribed genes are needed to more accurately estimate the frequency of gene loss in X. laevis after WGD.

Our comparative maps of functional genes between $X$. tropicalis and $X$. laevis demonstrated no evidence of interchromosomal rearrangements between two species and revealed that genetic linkage has been highly conserved between $X$. tropicalis and X. laevis except for inversions between XTR10 and homoeologous XLA15 and XLA18 pairs. The gene orders on $X$. tropicalis chromosomes have also been highly conserved in two homoeologous pairs of X. laevis chromosomes except for inversions between XLA12 and XLA16 and between XLA11 and XLA14. According to the gene orders of XTR3 and XTR8, which are considered to be the ancestral types of homoeologous XLA12 and XLA16 pairs and XLA11 and XLA14 pairs, respectively, a large paracentric inversion may have occurred in the long arm of 
Table 1 List of 60 genes localized to $X$. laevis chromosomes

\begin{tabular}{|c|c|c|c|c|c|}
\hline \multirow[t]{2}{*}{ Gene symbo/ } & \multirow[t]{2}{*}{ Clone no. ${ }^{\mathrm{b}}$ or accession no. } & \multicolumn{4}{|c|}{ Chromosomal location } \\
\hline & & $X$. laevis & $X$. tropicalis $^{\mathrm{C}}$ & Chicken ${ }^{d}$ & Human $^{\mathrm{d}}$ \\
\hline KDM3A & XL413k20ex & $1 p, 2 p$ & $1 p$ & $4 q$ & $2 p 11.2$ \\
\hline$A C S L 1$ & XL318g05ex & $1 p, 2 p$ & $1 p$ & $4 q$ & $4 q 34-q 35$ \\
\hline PCDH1O & XL003c24 & $1 p, 2 p$ & $1 p$ & $4 q$ & $4 q 28.3$ \\
\hline EEF2 & XL470I07ex & $1 q, 2 q$ & $1 q$ & 28 & 19pter-q12 \\
\hline$D M R T 1^{\mathrm{e}}$ & AB201112 & $1 q, 2 q$ & $1 q$ & $\mathrm{Zq}$ & $9 p 24.3$ \\
\hline NF2 & XL085b04 & $1 q, 2 q$ & $1 q$ & 15 & $22 q 12.2$ \\
\hline$D E P D C 1 B$ & XL220a24 & $1 q, 2 q$ & $1 q$ & $\mathrm{Zp}$ & $5 q 12.1$ \\
\hline POU2F1 & XL164e23 & $3 p, 8 p$ & $2 p$ & $1 q$ & 1q22-q23 \\
\hline EIF2S3 & XL408e08ex & $3 p, 8 p$ & $2 p$ & $1 q$ & Xp22.2-p22.1 \\
\hline $\mathrm{MDH} 2$ & XL165g11 & $3 q, 8 q$ & $2 q$ & 19 & 7cen-q22 \\
\hline LARP4 & XL014a12 & $3 q, 8 q$ & $2 q$ & un & $12 q 13.12$ \\
\hline$R A B 6 A$ & XL038d18 & $3 q, 8 q$ & $2 q$ & $1 q$ & $11 q 13.3$ \\
\hline PPFIBP1 & XL062p09 & $12 p, 16 p$ & $3 p$ & $1 p$ & 12p11.23-p11.22 \\
\hline IK & XL168o22 & $12 q$ & $3 q$ & 13 & $5 q 31.3$ \\
\hline CSNK1A1 & XL221g09ex & $12 q, 16 q$ & $3 q$ & 13 & $5 q 32$ \\
\hline NET1 & $\mathrm{XL227m13ex}$ & $12 q, 16 q$ & $3 q$ & $1 p$ & $10 p 15$ \\
\hline CYP19A1 & BC079750 & $12 q$ & $3 q$ & 10 & $15 q 21.1$ \\
\hline XPO7 & XL214I11 & $12 q, 16 q$ & $3 q$ & 22 & 8p21 \\
\hline$W T 1^{\mathrm{e}}$ & D82051 & $13 p, 17 p$ & $4 p$ & $5 p$ & $11 \mathrm{p} 13$ \\
\hline$E D C 4$ & XL473f03ex & $13 p, 17 p$ & $4 p$ & 11 & $16 q 22.1$ \\
\hline KARS & $\mathrm{XL480m02ex}$ & $13 q, 17 q$ & $4 q$ & 11 & $16 q 23-q 24$ \\
\hline UAP1 & XL086p04 & $13 q, 17 q$ & $4 q$ & $8 p$ & 1q23.3 \\
\hline$A L A S 1$ & XL051o04 & $13 q, 17 q$ & $4 q$ & 12 & $3 p 21.1$ \\
\hline$N V L$ & XL479c13ex & $4 p$ & $5 p$ & $3 q$ & $1 q 41-q 42.2$ \\
\hline XPO1 & XL294p07ex & $4 p, 5 p$ & $5 p$ & $3 p$ & $2 \mathrm{p} 16$ \\
\hline CEBPZ & XL039I04 & $4 p, 5 p$ & $5 p$ & $3 q$ & $2 p 22.2$ \\
\hline$S L C 2 A 12$ & XL036o21 & $4 q$ & $5 q$ & $3 q$ & $6 q 23.2$ \\
\hline TRIP12 & XL204I01 & $4 q, 5 q$ & $5 q$ & 9 & $2 q 36.3$ \\
\hline GATA4 & XL039m17 & $4 q, 5 q$ & $5 q$ & $3 q$ & 8p23.1-p22 \\
\hline$A B C F 2$ & XL012I18 & $6 p, 9 p$ & $6 p$ & $2 p$ & $7 q 36$ \\
\hline$W A C$ & XL075d07 & $6 p, 9 p$ & $6 p$ & $2 p$ & $10 p 11.2$ \\
\hline CTNNB1 & XL480g03ex & $6 p, 9 p$ & $6 p$ & $2 p$ & $3 p 21$ \\
\hline$A P C D D 1$ & XL055h12 & $6 q, 9 q$ & $6 q$ & $2 q$ & $18 p 11.22$ \\
\hline$E E F 1 D$ & XL013c21 & $6 q, 9 q$ & $6 q$ & $2 q$ & $8 q 24.3$ \\
\hline SEC23IP & XL151i10 & $7 p, 10 p$ & $7 p$ & 6 & $10 q 25-q 26$ \\
\hline ZRANB1 & XL027a17 & $7 p, 10 p$ & $7 p$ & 6 & $10 q 26.13$ \\
\hline CYP17A1 & AF325435 & $7 p$ & $7 p$ & 6 & $10 q 24$ \\
\hline GOT1 & XL151j11 & $10 p$ & $7 p$ & 6 & 10q24 \\
\hline TUBGCP2 & XL008b12 & $7 p$ & $7 p$ & 6 & $10 q 26.3$ \\
\hline SLC37A2 & XL082p15 & $7 q, 10 q$ & $7 q$ & 24 & $11 q 24.2$ \\
\hline$Z W 10$ & XL046I12 & $7 q, 10 q$ & $7 q$ & 24 & $11 q 23.2$ \\
\hline DDOST & XL005p17 & $7 q, 10 q$ & $7 q$ & 21 & 1p36.1 \\
\hline$G S N$ & XL300b05ex & $11 p, 14 p$ & $8 p$ & 17 & 9q33 \\
\hline$N R 5 A 1^{\mathrm{e}}$ & AB273177 & $14 p$ & $8 q$ & 17 & 9q33 \\
\hline$A R^{e}$ & U67129 & $14 q$ & $8 q$ & $4 p$ & Xq11.2-q12 \\
\hline FMR1 & XL022n18 & $11 q, 14 q$ & $8 q$ & $4 p$ & Xq27.3 \\
\hline sox3e & $-^{f}$ & $11 q$ & $8 q$ & $4 p$ & Xq27.1 \\
\hline PAPOLA & XL035j18 & $11 p, 14 q$ & $8 q$ & $5 q$ & $14 q 32.31$ \\
\hline ACTN1 & XL286g19ex & $11 p, 14 q$ & $8 q$ & $5 q$ & $14 q 22-q 24$ \\
\hline COPA & XL263c24ex & $11 q, 14 q$ & $8 q$ & 25 & 1q23-q25 \\
\hline STAU1 & XL175g01 & $15 p, 18 p$ & $10 q$ & 20 & $20 q 13.1$ \\
\hline$B M P 7$ & XL056I08 & $15 p, 18 p$ & $10 p$ & 20 & $20 q 13$ \\
\hline EFTUD2 & XL300a14ex & $15 p, 18 p$ & $10 p$ & 27 & $17 q 21.31$ \\
\hline soxge & AB439583 & $15 p, 18 p$ & $10 q$ & 18 & $17 q 24.3-q 25.1$ \\
\hline$N A R F$ & XL210h06 & $15 q, 18 q$ & $10 q$ & 18 & $17 q 25.3$ \\
\hline ZEB2 & XL207j23 & $15 q, 18 q$ & $9 q$ & $7 q$ & $2 q 22$ \\
\hline FN1 & XL338p06ex & $15 q, 18 q$ & $9 q$ & $7 p$ & $2 q 34$ \\
\hline
\end{tabular}


Table 1 (Continued)

Gene symbop

Clone no. ${ }^{\mathrm{b}}$ or accession no.

Chromosomal location

\begin{tabular}{|c|c|c|c|c|c|}
\hline & & X. laevis & X. tropicalis & Chicken $^{\mathrm{d}}$ & Humand \\
\hline NDUFS 1 & XL034k12 & $15 q, 18 q$ & $9 q$ & $7 q$ & $2 q 33-q 34$ \\
\hline NOMO3 & XL271k19ex & $15 q, 18 q$ & $9 p$ & 14 & $16 p 13$ \\
\hline UQCRC2 & XL016d01 & $15 q, 18 q$ & $9 p$ & 14 & $16 p 12$ \\
\hline
\end{tabular}

Abbreviations: EST, expressed sequence tag; un, unknown chromosomal location.

aHuman gene symbol.

${ }^{b}$ Clone numbers of $X$. laevis EST clones used for mapping, which were selected from a web data catalog of the NIBB/NIG/NBRP Xenopus laevis EST project (XDB3, http://xenopus. nibb.ac.jp/) based on the $X$. tropicalis chromosome map described by Uno et al. (2012). Fragment sizes of all $X$. laevis EST clones were $>1.5 \mathrm{~kb}$.

${ }^{c}$ Chromosomal locations for $X$. tropicalis taken from previous studies (Uno et al., 2008, 2012).

dChromosomal locations of chicken and human homologs searched with the BLATN program of the Ensembl (http://www.ensembl.org/index.html) and/or the blastn program of NCBI (http:// www.ncbi.nlm.nih.gov/) (searched in September 2012).

e'Genes mapped in our previous studies (Uno et al., 2008; Yoshimoto et al., 2008).

The cDNA fragment of SOX3 was isolated by Koyano et al. (1997).
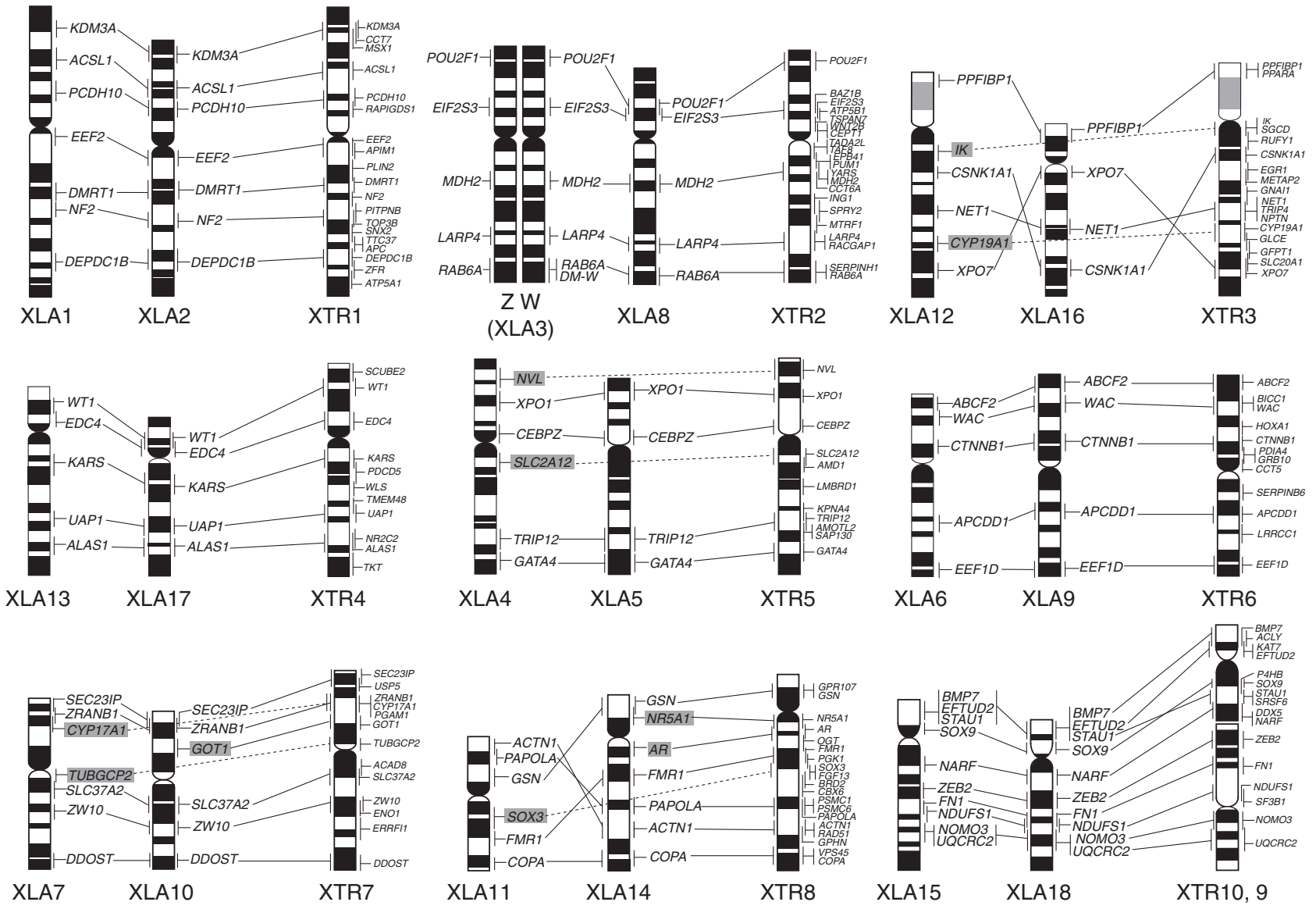

Figure 3 Comparative cytogenetic map of $X$. laevis (XLA) and $X$. tropicalis (XTR). The chromosomal locations of AR, CYP17A1, CYP19A1, DMRT1, DM-W, NR5A1, SOX3, SOX9 and WT1 in X. laevis and the cytogenetic map of $X$. tropicalis were taken from our previous studies (Uno et al., 2008; Yoshimoto et al., 2008). XTR9 is inverted to facilitate a comparison of the gene order with those of XLA15 and XLA18. Gene symbols enclosed in gray boxes indicate the genes that were located on only one pair of homoeologous chromosomes. The small size variations of XLA12p and XTR3p between individuals are represented as gray-colored bands in the ideogram (Figure 1; Uno et al., 2008).

XLA16, with at least two inversions containing a pericentric inversion in XLA11. The gene orders of SOX9 and STAU1 and their locations in XTR10q are different from those in XLA15p and XLA18p, suggesting that a pericentric inversion event occurred in the proximal region of the ancestral chromosome of homoeologous XLA15 and XLA18 pairs after the fusion between XTR9 and XTR10 of the ancestral diploid species such as $X$. tropicalis. These results provide us with the following two possibilities: (1) gene orders were different in some chromosomes between the ancestral diploid species before WGD occurred; and (2) intrachromosomal rearrangements occurred in one of the homoeologous chromosome pairs after WGD. In either case, the present results indicate that inter- and/or intrachromosomal rearrangements occurred much less in the $X$. laevis lineage for 21-40 million years after allotetraploidization than in teleost fishes 
(medaka fish, Takifugu, Tetraodon and zebrafish) (Kasahara et al., 2007; Sémon and Wolfe, 2007), salmonid fishes (Danzmann et al., 2008; Guyomard et al., 2012), plants (Arabidopsis, rice and Populus) (Pontes et al., 2004; Simillion et al., 2004; Yu et al., 2005; Tuskan et al., 2006) and yeast (Seoighe and Wolfe, 1998; Fischer et al., 2006), in which chromosome rearrangements frequently occurred after WGDs.

Neither multivalent association nor pairing between homoeologous chromosomes was observed in meiosis in X. laevis (Tymowska, 1991), which implies that an ancient allotetraploid genome of $X$. laevis returned to a genetically diploid state through the process of diploidization of allopolyploid genomes. The present results consequently suggest that the loss of duplicated genes and chromosomal rearrangements may not have been essential for diploidization of the allotetraploid genome after WGD in X. laevis. In highpolyploid Xenopus species with 72 and 108 chromosomes, a few multivalents have been observed at the first meiotic metaphase of some spermatocytes in $X$. vestitus, $X$. wittei, $X$. amieti $(2 n=72)$ and $X$. ruwenzoriensis $(2 n=108)$, suggesting that higher polyploidization may have been the most recent event in the Xenopus lineage and diploidization has yet not been fully accomplished (Tymowska and Fischberg, 1973; Tymowska, 1991).

In this study, we constructed a comparative cytogenetic map of the allotetraploid species $X$. laevis. The genetic linkage and order of genes have been highly conserved between $X$. tropicalis and $X$. laevis chromosomes and also between homoeologous chromosomes of $X$. laevis, and WGD-derived duplicated genes have been mostly retained in homoeologous chromosomes of $X$. laevis. These results collectively suggest that inter- and intrachromosomal rearrangements and loss of duplicated genes have occurred less frequently in the lineage of $X$. laevis after allotetraploidization. Whole-genome sequencing of $X$. laevis and comparative chromosome mapping of other polyploid Xenopus species help us to better understand the process and mechanism of genome evolution after WGD.

\section{DATA ARCHIVING}

There were no data to deposit.

\section{CONFLICT OF INTEREST}

The authors declare no conflict of interest.

\section{ACKNOWLEDGEMENTS}

This study was supported by a Grant-in-Aid for Scientific Research on Innovative Areas (No. 23113004) and a Grant-in-Aid for Scientific Research (B) (No. 22370081) from the Ministry of Education, Culture, Sports, Science and Technology (MEXT), Japan.

Allendorf FW, Thorgaard GH (1984). Tetraploidy and the evolution of salmonid fishes. In: Turner BJ (ed.) Evolutionary Genetics of Fishes. Plenum Press: New York, pp 1-53.

Bisbee CA, Baker MA, Wilson AC, Haji-Azumi I, Fischberg M (1977). Albumin phylogeny for clawed frogs (Xenopus). Science 195: 785-787.

Bowers JE, Chapman BA, Rong J, Paterson AH (2003). Unravelling angiosperm genome evolution by phylogenetic analysis of chromosomal duplication events. Nature 422 433-438.

Chain FJJ, Evans BJ (2006). Multiple mechanisms promote the retained expression of gene duplicates in the tetraploid frog Xenopus laevis. PLoS Genet 2: e56.

Danzmann RG, Davidson EA, Ferguson MM, Gharbi K, Koop BF, Hoyheim B et al. (2008), Distribution of ancestral proto-Actinopterygian chromosome arms within the genomes of $4 \mathrm{R}$-derivative salmonid fishes (Rainbow trout and Atlantic salmon). BMC Genomics 9: 557

Estes R (1975). Xenopus from the Palaeocene of Brazil and its zoogeographic importance. Nature 254: 48-50.

Evans BJ, Kelley DB, Tinsley RC, Melnick DJ, Cannatella DC (2004). A mitochondrial DNA phylogeny of African clawed frogs: phylogeography and implications for polyploid evolution. Mol Phylogenet Evol 33: 197-213.
Evans BJ (2008). Genome evolution and speciation genetics of clawed frogs (Xenopus and Silurana). Front Biosci 13: 4687-4706.

Fischer G, Rocha EP, Brunet F, Vergassola M, Dujon B (2006). Highly variable rates of genome rearrangements between hemiascomycetous yeast lineages. PLoS Genet 2: e32.

Guyomard R, Boussaha M, Krieg F, Hervet C, Quillet E (2012). A synthetic rainbow trout linkage map provides new insights into the salmonid whole genome duplication and the conservation of synteny among teleosts. BMC Genet 13: 15 .

Hellsten U, Khokha MK, Grammer TC, Harland RM, Richardson P, Rokhsar DS (2007). Accelerated gene evolution and subfunctionalization in the pseudotetraploid frog Xenopus laevis. BMC Biol 5: 31

Hellsten U, Harland RM, Gilchrist MJ, Hendrix D, Jurka J, Kapitonov V et al. (2010). The genome of the western clawed frog Xenopus tropicalis. Science 328: 633-636.

Hughes MK, Hughes AL (1993). Evolution of duplicate genes in a tetraploid animal, Xenopus laevis. Mol Biol Evol 10: 1360-1369.

International Human Genome Sequencing Consortium (2001). Initial sequencing and analysis of the human genome. Nature 409: 860-921.

Jaillon O, Aury JM, Brunet F, Petit JL, Stange-Thomann N, Mauceli E et al. (2004). Genome duplication in the teleost fish Tetraodon nigroviridis reveals the early vertebrate proto-karyotype. Nature 431: 946-957.

Kasahara M (2007). The 2R hypothesis: an update. Curr Opin Immunol 19: 547-552.

Kasahara M, Naruse K, Sasaki S, Nakatani Y, Qu W, Ahsan B et al. (2007). The medaka draft genome and insights into vertebrate genome evolution. Nature 447: 714-719.

Kellis M, Birren BW, Lander ES (2004). Proof and evolutionary analysis of ancient genome duplication in the yeast Saccharomyces cerevisiae. Nature 428: 617-624.

Kobel HR (1996). Allopolyploid speciation. In: Tinsley RC, Kobel HR (eds) The Biology of Xenopus. Clarendon Press: Oxford, pp 391-401.

Koyano S, Ito M, Takamatsu N, Takiguchi S, Shiba T (1997). The Xenopus Sox3 gene expressed in oocytes of early stages. Gene 188: 101-107.

Krylov V, Kubickova S, Rubes J, Macha J, Tlapakova T, Seifertova E et al. (2010). Preparation of Xenopus tropicalis whole chromosome painting probes using lase microdissection and reconstruction of $X$. laevis tetraploid karyotype by Zoo-FISH. Chromosome Res 18: 431-439.

Matsuda Y, Chapman VM (1995). Application of fluorescence in situ hybridization in genome analysis of the mouse. Electrophoresis 16: 261-272.

Morin RD, Chang E, Petrescu A, Liao N, Griffith M, Chow W et al. (2006). Sequencing and analysis of 10,967 full-length cDNA clones from Xenopus laevis and Xenopus tropicalis reveals post-tetraploidization transcriptome remodeling. Genome Res 16: 796-803.

Ohno S (1970). Evolution by Gene Duplication. Springer-Verlag: Berlin.

Otto SP (2007). The evolutionary consequences of polyploidy. Cell 131: 452-462.

Pontes O, Neves N, Silva M, Lewis MS, Madlung A, Comai L et al. (2004). Chromosoma locus rearrangements are a rapid response to formation of the allotetraploid Arabidopsis suecica genome. Proc Natl Acad Sci USA 101: 18240-18245.

Putnam NH, Butts T, Ferrier DEK, Furlong RF, Hellsten U, Kawashima T et al. (2008). The amphioxus genome and the evolution of the chordate karyotype. Nature 453: 1064-1071.

Sato Y, Hashiguchi Y, Nishida M (2009). Temporal pattern of loss/persistence of duplicate genes involved in signal transduction and metabolic pathways after teleost-specific genome duplication. BMC Evol Biol 9: 127

Schmid M, Steinlein C (1991). Chromosome banding in Amphibia. XVI. High-resolution replication banding patterns in Xenopus laevis. Chromosoma 101: 123-132.

Sémon M, Wolfe KH (2007). Rearrangement rate following the whole-genome duplication in teleosts. Mol Biol Evol 24: 860-867.

Sémon M, Wolfe KH (2008). Preferential subfunctionalization of slow-evolving genes after allopolyploidization in Xenopus laevis. Proc Natl Acad Sci USA 105: 8333-8338.

Seoighe C, Wolfe KH (1998). Extent of genomic rearrangement after genome duplication in yeast. Proc Natl Acad Sci USA 95: 4447-4452.

Simillion C, Vandepoele K, Saeys Y, Van de Peer Y (2004). Building genomic profiles for uncovering segmental homology in the twilight zone. Genome Res 14: 1095-1106.

Song K, Lu P, Tang K, Osborn TC (1995). Rapid genome change in synthetic polyploids of Brassica and its implications for polyploid evolution. Proc Natl Acad Sci USA 92 7719-7723.

Tuskan GA, Difazio S, Jansson S, Bohlmann J, Grigoriev I, Hellsten U et al. (2006). The genome of black cottonwood, Populus trichocarpa (Torr. \& Gray). Science $\mathbf{3 1 3}$ 1596-1604.

Tymowska J (1991). Polyploidy and cytogenetic variation in frogs of the genus Xenopus. In Green DS, Sessions SK (eds) Amphibian Cytogenetics and Evolution. Academic Press: San Diego, pp 259-297.

Tymowska J, Fischberg M (1973). Chromosome complements of the genus Xenopus. Chromosoma 44: 335-342.

Uno Y, Nishida C, Yoshimoto S, Ito M, Oshima Y, Yokoyama S et al. (2008). Diversity in the origins of sex chromosomes in anurans inferred from comparative mapping of sexual differentiation genes for three species of the Raninae and Xenopodinae. Chromosome Res 16: 999-1011.

Uno Y, Nishida C, Tarui H, Ishishita S, Takagi C, Nishimura 0 et al. (2012). Inference of the protokaryotypes of amniotes and tetrapods and the evolutionary processes of microchromosomes from comparative gene mapping. PLoS One 7: e53027.

Woodhouse MR, Schnable JC, Pedersen BS, Lyons E, Lisch D, Subramaniam S et al. (2010). Following tetraploidy in maize, a short deletion mechanism removed genes preferentially from one of the two homologs. PLoS Biol 8: e1000409.

Yoshimoto S, Okada E, Umemoto H, Tamura K, Uno Y, Nishida-Umehara C et al. (2008). A W-linked DM-domain gene, $D M-W$, participates in primary ovary development in Xenopus laevis. Proc Natl Acad Sci USA 105: 2469-2474.

Yu J, Wang J, Lin W, Li S, Li H, Zhou J et al. (2005). The genomes of Oryza sativa: a history of duplications. PLOS Biol 3: e38. 